\title{
LTV Model Reductions with Upper Error Bounds
}

\author{
Anders Helmersson \\ Division of Automatic Control \\ Department of Electrical Engineering \\ Linköpings universitet, SE-581 83 Linköping, Sweden \\ WWW: http://www. control.isy.liu.se \\ E-mail: andersh@isy.liu.se
}

1st February 2006

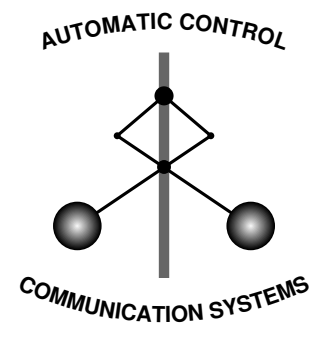

LINKÖPING

Report no.: LiTH-ISY-R-2725

Submitted to Transactions on Automatic Control

Technical reports from the Control \& Communication group in Linköping are available at http://www.control.isy.liu.se/publications. 



\begin{abstract}
An approach for computing upper error bounds for reduced-order models of linear time-varying systems is presented. It is based on a transformation technique of the Hankel singular values using positive-real, odd incremented functions. By applying such time-varying functions, the singular values to be removed can be forced to become equal and constant, so that they can be reduced in one step. Two variations of this method are proposed: one for finite-time horizons and the other for infinite-time problems.
\end{abstract}

Keywords: Rational interpolation, positive real functions, model reduction, linear time-varying systems. 



\title{
LTV Model Reduction with Upper Error
}

\section{Bounds}

\author{
Anders Helmersson \\ Department of Electrical Engineering \\ Linköping University \\ SE-581 83 Linköping, Sweden \\ www: http: //www. control.isy.liu.se \\ email: andersh@isy.liu.se
}

\begin{abstract}
An approach for computing upper error bounds for reduced-order models of linear time-varying systems is presented. It is based on a transformation technique of the Hankel singular values using positive-real, odd incremented functions. By applying such time-varying functions, the singular values to be removed can be forced to become equal and constant, so that they can be reduced in one step. Two variations of this method are proposed: one for finite-time horizons and the other for infinite-time problems.
\end{abstract}

\section{Index Terms}

Rational interpolation, positive real functions, model reduction, linear time-varying systems.

\section{INTRODUCTION}

Model reduction is an important tool when modeling dynamic systems and designing controllers. This paper considers model reduction of linear time-varying (LTV) systems and related error bounds. Both continuous and discrete time systems will be covered in a uniform way.

\footnotetext{
${ }^{0}$ Source: mred.tex 180 2006-01-25 13:52:29Z andersh.
} 
It is well-known that Lyapunov equations defining observability and reachability Gramians play an important role when making model reduction and estimating error bounds [1], [2], [3], [4], [5]. Using these Gramians we can find a state transformation that makes both Gramians equal and diagonal in the new, balanced state space. The elements in the balanced Gramian are the Hankel singular values (HSV).

In the linear time-invariant (LTI) case we can perform model reduction such that the model error becomes less or equal to the sum of the tail of removed Hankel singular values. If two or more singular values are equal they need to be counted only once.

By modifying the Lyapunov equations to become linear matrix inequalities (LMIs) the result above also applies and in addition provides more flexibility in the analysis [6], [7]. In this case it is also well-known that if a reduced model of order $r$ exists then we can always find a generalized balanced Gramian such that the $n-r$ smallest singular values become equal, where $n$ is the order of the original system [8], [9], [10].

This paper focuses on how to find upper error bounds for a reduced order model based on the knowledge of the Hankel singular values only. The analysis is based on three observations:

- If one or more Hankel singular values are equal they can be reduced in one step with a model error of that singular value. If LTV systems are considered they need to be equal and constant over time.

- If there exists a reduced model of order $r<n$ with an error bound of $\gamma$, then there exists a generalized balanced realization such that the $n-r$ smallest Hankel singular values become equal to $\gamma$ and constant over time [10].

- If $\Sigma>0$ defines the Hankel singular values and if $\alpha \geq 0$ then also $\bar{\Sigma}=\Sigma+\alpha \Sigma^{-1}$ are generalized Hankel singular values for the system. This holds for both discrete and continuous time LTV systems.

The last observation, which is related to convex invertible cones [11], is the main tool in this paper. It can be used to make several Hankel singular values to become equal. For instance using $\alpha=\sigma_{n} \sigma_{n-1}$ we can obtain a modified balanced Gramian, $\bar{\Sigma}$, in which the two smallest Hankel singular become equal to $\sigma_{n}+\sigma_{n-1}$. By using the modification recursively we can force the $n-r$ smallest singular values to become equal. The modification of the balanced Gramian is performed using a particular transformation, $W$, which is characterized as $W(x)-x$ being a positive-real, odd, analytic function. This fact allows us to employ results from interpolation 
theory of positive-real functions.

Note that this analysis is performed based on the Hankel singular values only, without any explicit knowledge of the $A, B$, and $C$ matrices. The only assumption is that the Hankel singular values define the balanced Gramian satisfying the observability and reachability Lyapunov inequalities.

In the time-varying case, similar time-varying functions or, transformations, can be used. Here we need to add monotonicity requirements on the transformation, which means that the function used to modify the reachability Gramian must be increasing, at least for all Hankel singular values. Similarly the transformation used to modify the observability Gramian must be decreasing.

We will show how to construct such monotonic transformations in order to force the last $n-r$ Hankel singular values to become equal and constant. The results can be condensed into model error bounds that depend on the sum of maximum Hankel singular values and the number of minima. The model error will increase gracefully, approximately as the logarithm of the number of minima of the Hankel singular values.

We can also provide bounds that are independent of the number of minima and time horizon. For instance, when reducing an LTV system to any order the model error is always bounded by twice the maximum value of the sum of all Hankel singular values. In general, this type of bound depends not only on the removed Hankel singular values but also on remaining ones.

The upper error bounds presented in this paper are in most cases conservative, even if only the the Hankel singular values are available. Based on some specific Hankel singular values as function of time, we can probably design a tailored transformation that provides a better upper bound. The upper error bounds presented here are compromises between simplicity and accuracy.

\section{A. Notation}

The notation used in this paper is fairly standard. The sets of real and complex numbers are denoted by $\mathbb{R}$ and $\mathbb{C}$ respectively. The open set of complex numbers with strictly positive real part is denoted by $\mathbb{C}_{+}$and the corresponding closed set by $\overline{\mathbb{C}}_{+}$. We denote an identity matrix of size $m$ by $I_{m}$. When clear from the context, the subindex will be omitted. For a square, symmetric (Hermitian) matrix, $A>(\geq) 0$ denotes a positive (semi-)definite matrix. 
Hankel singular values including their generalized versions, are denoted by $\sigma_{i}$. The balanced Gramian, $\Sigma=\operatorname{diag}\left[\sigma_{1}, \ldots, \sigma_{n}\right]$ is assumed to be ordered in descending order. We split $\Sigma$ into two block diagonal parts, where $\Sigma_{R}$ contains the Hankel singular values that correspond to the remaining states after model reduction and $\Sigma_{N}$ corresponds to the removed states. We also use $\Sigma_{R}$ and $\Sigma_{N}$ to denote the sets of Hankel singular values that they hold in their diagonals.

\section{Preliminaries}

We will here study the problem of approximating a discrete-time, stable, LTV system,

$$
G: u \rightarrow y, \quad \text { where } \begin{cases}x(t+1) & =A(t) x(t)+B(t) u(t) \\ y(t) & =C(t) x(t)+D(t) u(t)\end{cases}
$$

by a lower order system denoted $\hat{G}$. In the continuous-time case the system is described by

$$
G: u \rightarrow y, \text { where }\left\{\begin{array}{l}
\dot{x}(t)=A(t) x(t)+B(t) u(t) \\
y(t)=C(t) x(t)+D(t) u(t),
\end{array}\right.
$$

which also is assumed to be stable.

We will use the induced $\ell_{2}$ and $L_{2}$-norm as performance criterion, $\gamma=\|G-\hat{G}\|$.

\section{A. Gramians and Internal Balancing}

In discrete time, a generalized controllability or reachability Gramian P satisfies

$$
A(t) P(t) A^{T}(t)+B(t) B^{T}(t) \leq P(t+1)
$$

and a generalized observability Gramian $Q$

$$
A^{T}(t) Q(t+1) A(t)+C^{T}(t) C(t) \leq Q(t) .
$$

In continuous time, the corresponding generalized Gramians satisfy

$$
A(t) P(t)+P(t) A^{T}(t)-\dot{P}(t)+B(t) B^{T}(t) \leq 0,
$$

and

$$
Q(t) A(t)+A^{T} Q(t)+\dot{Q}(t)+C^{T}(t) C(t) \leq 0
$$

A similarity transformation does not change the input-output transfer function, but does change the Gramians. It is possible to find a similarity transformation, $\bar{x}=T x$, that makes 
the reachability and observability Gramians equal, $P=Q=\Sigma$, see for instance [12], [13] for details. In the discrete-time case, the balanced, generalized observability and reachability Gramians now satisfy

$$
\begin{aligned}
\bar{A}^{T}(t) \Sigma(t+1) \bar{A}(t)+\bar{C}^{T}(t) \bar{C}(t) & \leq \Sigma(t), \\
\bar{A}(t) \Sigma(t) \bar{A}^{T}(t)+\bar{B}(t) \bar{B}^{T}(t) & \leq \Sigma(t+1),
\end{aligned}
$$

respectively. In continuous time, the balanced, generalized observability and reachability Gramians satisfy

$$
\begin{aligned}
& \Sigma(t) \bar{A}(t)+\bar{A}^{T}(t) \Sigma(t)+\dot{\Sigma}+\bar{C}^{T}(t) \bar{C}(t) \leq 0 \\
& \bar{A}(t) \Sigma(t)+\bar{A}^{T}(t) \Sigma(t)-\dot{\Sigma}+\bar{B}(t) \bar{B}^{T}(t) \leq 0
\end{aligned}
$$

respectively. It is assumed that $\Sigma$ is a continuous function of time.

In the sequel we will often use the term Gramians to also denote generalized Gramians.

\section{B. Upper Bounds for LTI systems}

We will first review the problem of approximating a stable LTI system $G: u \rightarrow y$ of order $n$ by a lower order system denoted $\hat{G}: u \rightarrow \hat{y}$, which is assumed to have order $r<n$.

As a criterion for how well the approximation fits to the original transfer function the $\mathcal{H}_{\infty^{-}}$ norm is used:

$$
\gamma=\|G-\hat{G}\|_{\infty}
$$

The optimal Hankel norm reduction (see [4] for a comprehensive treatment) is based on the balanced realization. Another scheme called truncated model reduction also employs the balanced realization. The balanced Gramian is diagonal with the Hankel singular values $\sigma_{i}$ on the diagonal:

$$
\Sigma=\operatorname{diag}\left[\sigma_{1}, \sigma_{2}, \ldots, \sigma_{n}\right]
$$

Using the Hankel-norm model reduction defined in [4], the $\mathcal{H}_{\infty}$ model error $\gamma$ of an $r$ th order approximation is bounded by

$$
\gamma \leq \sum_{\substack{i=r+1 \\ \sigma_{i} \neq \sigma_{i+1}}}^{n} \sigma_{i}
$$


where the Hankel singular values $\sigma_{i}$ are assumed to be ordered

$$
\sigma_{1} \geq \sigma_{2} \geq \ldots \geq \sigma_{r} \geq \sigma_{r+1} \geq \ldots \geq \sigma_{n} \geq 0
$$

In (9) equal singular values are only added once. For any reduced model of order $r$ we have that $\gamma \geq \sigma_{r+1}$. Thus, the Hankel-norm model reduction is optimal in the $\mathcal{H}_{\infty}$ sense if $r=n-1$, but not necessarily so in the general case.

\section{Linear Time-Varying Model Reduction}

The results for the linear time-varying problem is similar to the time-invariant case. A important difference though, is that we here assume that the Hankel singular values to be removed are equal and constant over time. To simplify, it is also assumed that the $n-r$ smallest Hankel singular values are equal to one: $\sigma_{r+1}=\ldots=\sigma_{n}=1$, which can be obtained after a simple scaling of the inputs and outputs.

In the discrete-time case, it is then possible to find a reduced order system $\hat{G}$ such that $\|G-\hat{G}\| \leq 1$ :

$$
\left[\begin{array}{cc}
\hat{A}(t) & \hat{B}(t) \\
\hat{C}(t) & \hat{D}(t)-D(t)
\end{array}\right]=\left[\begin{array}{c|cc}
0 & \Gamma_{1}^{T}(t+1) & 0 \\
C(t) & 0 & 0
\end{array}\right]\left[\begin{array}{cc}
\Sigma(t) & -A^{T}(t) \\
-A(t) & \Sigma(t+1)
\end{array}\right]^{-1}\left[\begin{array}{cc}
\Gamma_{1}(t) & 0 \\
0 & 0 \\
\hline 0 & B(t)
\end{array}\right],
$$

where $\Gamma_{1}^{2}=\Sigma_{1}^{2}-I$ and $\Sigma_{1}=\operatorname{diag}\left[\sigma_{1}, \ldots, \sigma_{r}\right]$. Similarly, in continuous time [4] we can use

$$
\begin{aligned}
& {\left[\begin{array}{ll}
\hat{A} & \hat{B} \\
\hat{C} & \hat{D}
\end{array}\right]=\left[\begin{array}{cc}
\Gamma_{1}^{-1} & 0 \\
0 & I
\end{array}\right]\left\{\left[\begin{array}{cc}
\Sigma_{1} A_{11} \Sigma_{1}+A_{11}^{T} & \Sigma_{1} B_{1} \\
C_{1} \Sigma_{1} & D
\end{array}\right]\right.} \\
& \left.-\left[\begin{array}{c}
\Sigma_{1} A_{12}+A_{21}^{T} \\
C_{2}
\end{array}\right]\left(A_{22}+A_{22}^{T}\right)^{-1} \times\left[A_{21} \Sigma_{1}+A_{12}^{T} \quad B_{2}\right]\right\}\left[\begin{array}{cc}
\Gamma_{1}^{-1} & 0 \\
0 & I
\end{array}\right] .
\end{aligned}
$$

where the time dependency has been dropped.

For both the discrete and continuous time cases the remaining generalized Hankel singular values after the model reduction, $\Sigma_{1}$, satisfy the Lyapunov inequalities for the reduced system. This means that the reduced system is balanced with $\Sigma_{1}$ as Hankel singular values. 


\section{Balanced Truncation}

It is important to note that the reduced models defined in discrete (11) and continuous time (12), depend on the Hankel singular values, $\Sigma$. This is in contrast to balanced truncation, see for instance [14], which only depends on the original (truncated) $A, B$, and $C$ matrices; the $D$ matrix is always kept untouched:

$$
\left[\begin{array}{ll}
\hat{A} & \hat{B} \\
\hat{C} & \hat{D}
\end{array}\right]=\left[\begin{array}{cc}
A_{11} & B_{1} \\
C_{1} & D
\end{array}\right]
$$

This specifically means that the error bound can be assessed by removing one Hankel singular value at a time. After removing a state, we can make a fresh start using the original, truncated $\Sigma$ to assess the error bound for removing the next state, and so on. As we will later see, this will not be the case when using (11) and (12), since the Hankel singular values to be used in the next reduction step are modified. In addition, when using balanced truncation, the singular values can be orded arbitrarily. The drawback is that the model reduction error, when using (13) is $2 \sigma_{n}$, compared to $\sigma_{n}$ when using (11) and (12).

\section{TRANSFormations OF HANKEL Singular VALUES}

In the previous section we have seen that states can be removed with a model error equal to the smallest Hankel singular value, $\sigma_{n}$, provided it is constant in time. We will here consider some different schemes on how to modify $\Sigma$ based on the information that it satisfies (6) and (5) in the discrete-time case and (8) and (7) in the continuous time case, without any further knowledge of the specific $A, B$ and $C$. The aim is to modify the Hankel singular values so that the $n-r$ smallest singular values become equal and constant. Doing so, we can remove the corresponding states in one fell swoop, with a model error of $\gamma=\sigma_{r+1}=\ldots=\sigma_{n}$.

In order to characterize the set of allowable transformations on Hankel singular values (HSV) we start by the following definition in terms of a continued fraction expansion.

Definition 1 A rational HSV transformation function, $W_{r}(\sigma)$, of order $r$ is defined by

$$
W_{r}(z)=z+\alpha_{0} z+\alpha_{r}\left(z+\alpha_{r-1}\left(\ldots \alpha_{2}\left(z+\alpha_{1} \sigma^{-1}\right) \ldots\right)^{-1}\right)^{-1},
$$

where $\alpha_{i} \geq 0, \forall i \in[0, r]$. 
Assuming $\alpha_{0}=0$, we can also rewrite this as an recursive definition with $W_{0}(z)=z$ and $W_{r}(z)=z+\alpha_{r} W_{r-1}^{-1}(z)$ for $r \geq 0$.

\section{A. Basic Properties}

Lemma 1: Let $W_{r}$ be a HSV transformation function according to Definition 1. If $\Sigma>0$ satisfies the observability and reachability Lyapunov inequalities,

$$
\begin{aligned}
A^{T}(t) \Sigma(t+1) A(t)+C^{T}(t) C(t) & \leq \Sigma(t), \\
A(t) \Sigma(t) A^{T}(t)+B(t) B^{T}(t) & \leq \Sigma(t+1),
\end{aligned}
$$

in the discrete-time case, and

$$
\begin{aligned}
& \Sigma(t) A(t)+A(t)^{T} \Sigma(t)+\dot{\Sigma}(t)+C^{T}(t) C(t) \leq 0 \\
& A(t) \Sigma(t)+\Sigma(t) A^{T}(t)-\dot{\Sigma}(t)+B(t) B^{T}(t) \leq 0
\end{aligned}
$$

in the continuous-time case, then also any $\bar{\Sigma}=W_{r}(\Sigma)$ does so for any $\alpha_{i} \geq 0$.

Proof: We show this by induction. First, by assumption $W_{0}(\Sigma)=\Sigma$ satisfies (15) and (16). Secondly, assuming that $\bar{\Sigma}=W_{r}(\Sigma)$ satisfies (15) implies $A^{T}(t) W_{r}(\Sigma(t+1)) A(t) \leq W_{r}(\Sigma(t))$. Since $W_{r}(\Sigma(t+1)) \geq \Sigma(t+1)>0$, we can use Schur complement to rewrite this as

$$
\left[\begin{array}{cc}
-W_{r}(\Sigma(t)) & A^{T}(t) \\
A(t) & -W_{r}^{-1}(\Sigma(t+1))
\end{array}\right] \leq 0,
$$

which in turn is equivalent to $A(t) W_{r}^{-1}(\Sigma(t)) A^{T}(t) \leq W_{r}^{-1}\left(\Sigma(t+1)\right.$, since $W_{r}(\Sigma(t)) \geq \Sigma(t)>$ 0 . Combining this with (15) yields

$$
A(t) W_{r+1}(\Sigma(t)) A^{T}(t)+B(t) B^{T}(t) \leq W_{r+1}(\Sigma(t+1)) .
$$

where $W_{r+1}(\Sigma)=\Sigma+\alpha_{r+1} W_{r}^{-1}(\Sigma)$ and $\alpha_{r+1} \geq 0$. Analogously, we can show that,

$$
A^{T}(t) W_{r+1}(\Sigma(t+1)) A(t)+C^{T}(t) C(t) \leq W_{r+1}(\Sigma(t)),
$$

assuming $\bar{\Sigma}=W_{r}(\Sigma)$ satisfies (16), which completes the proof by induction. The continuoustime part of the Lemma is proved similarly. Removing the $B B^{T}$ from (18) and then multiplying it by $\Sigma^{-1}$ from the left and from the right yields

$$
\begin{aligned}
& \Sigma(t)^{-1} A(t)+A^{T}(t) \Sigma(t)^{-1}-\Sigma(t)^{-1} \dot{\Sigma}(t) \Sigma(t)^{-1} \\
& =\Sigma(t)^{-1} A(t)+A^{T}(t) \Sigma(t)^{-1}+\frac{d}{d t}\left(\Sigma(t)^{-1}\right) \leq 0,
\end{aligned}
$$

and consequently $\Sigma+\alpha \Sigma^{-1}$ also satisfies (17). 


\section{B. Positive-Real Functions}

We will now connect the HSV transformation to posivite real functions.

Definition 2 A function, $f: \mathbb{C} \rightarrow \mathbb{C}$, is positive real if it is analytic in $\mathbb{C}_{+}=\{z \in \mathbb{C}: \operatorname{Re} z>$ $0\}$, $\operatorname{Re} f(z) \geq 0$ for any $z \in \mathbb{C}_{+}$and $f(z) \in \mathbb{R}$ when $z \in \mathbb{R}$.

Lemma 2: Let $w=a / b$ be an odd, rational function, where $a$ and $b$ have no common factors. Then, the following statements are equivalent

(i) $w$ is on the form

$$
w(x)=\alpha_{0} x+\frac{\alpha_{1}}{x+\frac{\alpha_{2}}{\ddots}}, \quad \alpha_{i} \geq 0
$$

(ii) $w$ is positive real;

(iii) $a+b$ has all its zeros in $\mathbb{C}_{-}$.

Proof: (i) $\Rightarrow$ (ii) follows immediately from the recursive definition of $w$, since the sum of two positive-real functions are also positive real and since the inverse of a non-zero $r$ is also a positive-real function.

(ii) $\Rightarrow$ (iii) follows by the following arguments. Let $w=a / b$, where $a$ and $b$ are polynomial functions with no common zeros. Since $w$ is a positive-real function, it is analytic in $\overline{\mathbb{C}}_{+}$with the exception of single, isolated poles (zeros of $b$ ) on the imaginary axis. Using the principle of variation of argument we conclude that $a+b$ has no zeros in $\overline{\mathbb{C}}_{+}$with the exception of the singularities in $w$. Consequently all zeros of $a+b$ are located in the open left complex plane, $\mathbb{C}_{-}$.

(iii) $\Rightarrow$ (i) Using the standard Routh-Hurwitz algorithm, see [15, page 180], for determining stability of the polynomial,

$$
p(x)=a(x)+b(x)=a_{0} x^{n}+b_{0} x^{n-1}+a_{1} x^{n-2}+b_{1} x^{n-3}+\ldots
$$

we obtain the coefficients, $\alpha_{i}$ in $w$ as $\alpha_{0}=a_{0} / b_{0}, \alpha_{1}=c_{0} / b_{0}, \alpha_{2}=d_{0} / b_{0}, \alpha_{3}=e_{0} / c_{0}$, etc, where

$$
c_{i}=a_{i+1}-\frac{a_{0}}{b_{0}} b_{i+1}, \quad d_{i}=b_{i+1}-\frac{b_{0}}{c_{0}} c_{i+1}, \ldots
$$


are the coefficients in Routh's algorithm.

$$
w(x)=\frac{a_{0} x^{n}+a_{1} x^{n-2}+\ldots}{b_{0} x^{n-1}+b_{1} x^{n-3}+\ldots}=\frac{a_{0}}{b_{0}}\left(x+\frac{c_{0} / a_{0}}{x+\frac{d_{0} / b_{0}}{\ddots}}\right) .
$$

Since the characteristic polynomial, $a+b$, is Hurwitz, the coefficients, $\alpha_{i}$, are all positive.

Corollary 1: The rational function, $W_{r}$, is a HSV transformation if and only if $f(z)=W_{r}(z)-$ $z$ is a rational positive real, odd function.

The set of increments, $W_{r}(\Sigma)-\Sigma$, can also be described as the smallest convex invertible cone [11], [16] containing $\Sigma$.

Lemma 2 can be used to construct $\bar{\Sigma}=W_{r}(\Sigma)$ such that $r$ Hankel singular values (elements in the diagonal of $\bar{\Sigma}$ ) become equal.

Lemma 3: Let $r$ denote the number of strictly positive singular values, $\sigma_{i}>0$. Further let $q_{k}$ denote the coefficients in the characteristic polynomial of $\Sigma=\operatorname{diag}\left[\sigma_{1}, \sigma_{2}, \ldots, \sigma_{r}\right]$ defined by:

$$
p(x)=\prod_{k=1}^{r}\left(x+\sigma_{k}\right)=\sum_{k=0}^{r} q_{k} x^{k}
$$

Then

$$
W_{r}(x)=q_{r-1} \frac{x^{r}+q_{r-2} x^{r-2}+\ldots}{q_{r-1} x^{r-1}+q_{r-3} x^{r-3}+\ldots}=\frac{a(x)}{b(x)},
$$

is a HSV transformation function such that $W_{r}\left(\sigma_{i}\right)=\sum_{i=1}^{r} \sigma_{i}$.

Proof: According to Lemma 2, $p$ generates a positive-real function, $W_{r}$, that can be expressed as a continued fraction expansion yields:

$$
W_{r}(x)=x+\frac{\alpha_{1}}{x+\frac{\alpha_{2}}{x+\frac{\alpha_{2}}{\ddots}}},
$$

where $\alpha_{k} \geq 0$, which indeed is a HSV transformation.

\section{Derivation of Some Known Bounds}

In the time-invariant case, where $\sigma_{i}$ are constants with respect to time, we can remove the $n-r$ smallest Hankel singular values by using a HSV transformation, $W_{r}$, defined in terms of the removed singular values: $\sigma_{r+1}, \ldots, \sigma_{n}$. In this case, the model error is bounded by $\left\|G-\hat{G}_{r}\right\| \leq$ 
$\sum_{i=r+1}^{n} \sigma_{i}$. If there are multiple Hankel singular values, such that $\sigma_{i}=\sigma_{k}, i \neq k$, they only need to be counted once.

This is the same bound as if one singular value is removed at a time. After each removal the truncated Hankel singular values are valid for the reduced system. This means that the total error bound becomes equal to the sum of the removed singular values.

In the time-varying, discrete-time case with periodicity such that $\Sigma(t+T)=\Sigma(t)$ for all $t$ and for some $T$, the upper bound of the model error becomes

$$
\left\|G-\hat{G}_{r}\right\| \leq \sum_{t=1}^{T} \sum_{i=r+1}^{n} \sigma_{i}(t),
$$

using the HSV transformation defined by the set of Hankel singular values given by $\left\{\sigma_{i}(t)\right.$ : $i \in[r+1, n], t \in[1, T]\}$. Again, if there are multiple Hankel singular values, such that $\sigma_{i}(t)=\sigma_{k}(s),(i, t) \neq(k, s)$, they only need to be counted once. This bound has been previously presented for truncated systems, see [17], [18], [19], [14].

\section{Rational Interpolation}

According to Corollary 1 , the transformation function, $W_{r}$, as the property that $f(z)=$ $W_{r}(z)-z$ is a positive-real, odd, rational function. This allows us to employ results from analytic interpolation theory including the Nevanlinna-Pick problem: Given the interpolation data, $\left\{\left(z_{k}, w_{k}\right)\right\}_{k=1}^{n} \subset \mathbb{C}_{+} \times \mathbb{C}_{+}$, does there exist a positive-real function $f$ such that $f\left(z_{k}\right)=$ $w_{k}, k=1, \ldots, n$ ?

Lemma 4 (Nevanlinna-Pick): There exists a solution to the Nevanlinna-Pick interpolation problem, if and only if the Pick matrix

$$
P=\left[\begin{array}{cccc}
\frac{w_{1}+\bar{w}_{1}}{z_{1}+\bar{z}_{1}} & \frac{w_{1}+\bar{w}_{2}}{z_{1}+\bar{z}_{2}} & \ldots & \frac{w_{1}+\bar{w}_{n}}{z_{1}+\bar{z}_{n}} \\
\frac{w_{2}+\bar{w}_{1}}{z_{2}+\bar{z}_{1}} & \frac{w_{2}+\bar{w}_{2}}{z_{2}+\bar{z}_{2}} & \ldots & \frac{w_{2}+\bar{w}_{n}}{z_{2}+\bar{z}_{n}} \\
\vdots & \vdots & \ddots & \vdots \\
\frac{w_{n}+\bar{w}_{1}}{z_{n}+\bar{z}_{1}} & \frac{w_{n}+\bar{w}_{2}}{z_{n}+\bar{z}_{2}} & \ldots & \frac{w_{n}+\bar{w}_{n}}{z_{n}+\bar{z}_{n}}
\end{array}\right]
$$

is positive semidefinite. Here $\bar{z}$ denotes the complex conjugate of $z$.

The solution is unique if the Pick matrix, $P$, is singular, otherwise all solutions can be parametrized by a linear-fractional transformation.

The Pick matrix can be modified to also include derivative conditions of the type $f^{\prime}\left(z_{i}\right)=w_{i}^{\prime}$ (higher order derivatives can also be included). See for instance [20], [21] for details. 
As an alternative to the Pick matrix, Nevanlinna reduced the number of constraints using the Schur algorithm. If $f\left(z_{1}\right)=w_{1}$ then $f$ is positive real if and only if $f$ is on the form

$$
f(z)=w_{1} \frac{z_{1}+z f_{1}(z)}{z_{1} f_{1}(z)+z}
$$

where $f_{1}$ is also positive real. Note that (23) implies that we can always find an odd rational function if we assume that $\left\{\left(z_{k}, w_{k}\right)\right\} \in \mathbb{R}_{+} \times \mathbb{R}_{+}$.

\section{E. General Analytic Transformations}

So far we have restricted the HSV transformation functions to be rational functions. Using positive-real interpolation we can generalize Lemma 1.

Definition 3 [PROI function] A PROI function, $W(z)$, is an odd, analytic function such that $W(z)-z$ is positive-real

Lemma 5: Let $W$ be a PROI function as defined in Definition 3. If $\Sigma>0$ satisfies the observability and reachability Lyapunov inequalities, (15) and (16) in the discrete-time case, and (17) and (18) in the continuous-time case, then also any $\bar{\Sigma}=W(\Sigma)$ does so.

Proof: According to Lemmas 2 and 4, we can find a rational PROI function, $W_{r}$, such that $\bar{\Sigma}=W_{r}(\Sigma)=W(\Sigma)$ and $W_{r}^{\prime}(\Sigma)=W^{\prime}(\Sigma)$ hold. Thus, according to Lemma 1, also $\bar{\Sigma}$ satisfies the Lyapunov inequalities as $\Sigma$ does.

\section{F. Combining HSV transformations}

A HSV transformation (20), $W$, can also be written as

$$
W(x)=\bar{\sigma} \frac{p(x)+\bar{p}(x)}{p(x)-\bar{p}(x)}
$$

where $p(x)$ is defined in (19),

$$
\bar{p}(x)=\prod_{k=1}^{r}\left(x-\sigma_{k}\right)
$$

and $\bar{\sigma}=\sum_{i} \sigma_{i}$. In order to combine two HSV transformations, $W_{1}$ and $W_{2}$, so that the multiset union of their defining singular values become the singular values of the new function, we can use the following formula:

$$
W(x)=\left(\bar{\sigma}_{1}+\bar{\sigma}_{2}\right) \frac{W_{1}(x) W_{2}(x)+\bar{\sigma}_{1} \bar{\sigma}_{2}}{\bar{\sigma}_{2} W_{1}(x)+\bar{\sigma}_{1} W_{2}(x)}=\underbrace{\left(\bar{\sigma}_{1}+\bar{\sigma}_{2}\right)}_{\overline{\bar{\sigma}}}\left(\frac{W_{1}(x)}{\bar{\sigma}_{1}}\right) \oplus\left(\frac{W_{2}(x)}{\bar{\sigma}_{2}}\right),
$$


where the commutative and associative operator $\oplus$, is defined by

$$
x \oplus y=\frac{x y+1}{x+y} .
$$

Here we assume that $\bar{\sigma}_{i}=W_{i}\left(\sigma_{i}\right)$. This implies that

$$
W\left(\sigma_{1}\right)=W\left(\sigma_{2}\right)=\overline{\bar{\sigma}}=\bar{\sigma}_{1}+\bar{\sigma}_{2} .
$$

The general case with several singular values can be written as

$$
W(x)=\overline{\bar{\sigma}} \bigoplus_{i}\left(\frac{W_{i}(x)}{\bar{\sigma}_{i}}\right)=\overline{\bar{\sigma}} \frac{\prod_{i}\left(W_{i}(x)+\bar{\sigma}_{i}\right)+\prod_{i}\left(W_{i}(x)-\bar{\sigma}_{i}\right)}{\prod_{i}\left(W_{i}(x)+\bar{\sigma}_{i}\right)-\prod_{i}\left(W_{i}(x)-\bar{\sigma}_{i}\right)}
$$

where $\overline{\bar{\sigma}}=\sum_{i} \bar{\sigma}_{i}$. Taking the derivative of $W$ with respect to time assuming a fixed $x$ yields

$$
\begin{aligned}
\dot{W} & =\bar{\sigma}_{2} \frac{W_{1}\left(W_{1} W_{2}+2 \bar{\sigma}_{1} \bar{\sigma}_{2}+\bar{\sigma}_{2}^{2}-W_{2}^{2}\right)+\bar{\sigma}_{1}^{2} W_{2}}{\left(\bar{\sigma}_{2} W_{1}+\bar{\sigma}_{1} W_{2}\right)^{2}} \dot{W}_{1} \\
& +\bar{\sigma}_{1} \frac{W_{2}\left(W_{1} W_{2}+2 \bar{\sigma}_{1} \bar{\sigma}_{2}+\bar{\sigma}_{1}^{2}-W_{1}^{2}\right)+\bar{\sigma}_{2}^{2} W_{1}}{\left(\bar{\sigma}_{2} W_{1}+\bar{\sigma}_{1} W_{2}\right)^{2}} \dot{W}_{2} \\
& +\overline{\bar{\sigma}} \frac{\bar{\sigma}_{1}\left(W_{2}^{2}-\bar{\sigma}_{2}^{2}\right)}{\left(\bar{\sigma}_{2} W_{1}+\bar{\sigma}_{1} W_{2}\right)^{2}} \dot{\bar{\sigma}}_{1}+\overline{\bar{\sigma}} \frac{\bar{\sigma}_{2}\left(W_{1}^{2}-\bar{\sigma}_{1}^{2}\right)}{\left(\bar{\sigma}_{2} W_{1}+\bar{\sigma}_{1} W_{2}\right)^{2}} \dot{\bar{\sigma}}_{2} .
\end{aligned}
$$

Lemma 6: Let $\left(W_{i}, \bar{\sigma}_{i}\right) \in(\mathbb{R} \rightarrow \mathbb{R}) \times \mathbb{R}$ be $n$ pairs that satisfy the following properties:
(i) $W_{i} \geq x$
(iv) $\dot{W}_{i} \geq 0$,
(ii) $W_{i} \geq \bar{\sigma}_{i}$,
(v) $\bar{\sigma}_{i} \geq 0$, and
(iii) $x+\frac{\bar{\sigma}_{i}^{2}}{3 x} \geq W_{i}$,
(vi) $\dot{\bar{\sigma}}_{i} \geq 0$.

Then $W=\overline{\bar{\sigma}} \bigoplus_{i}\left(W_{i} / \sigma_{i}\right)$ and $\overline{\bar{\sigma}}=\sum_{i} \bar{\sigma}_{i}$ satisfy the very same properties.

Proof: We first verify the properties of $W$ when $n=2$. To show (i) of $W$, we can use

$$
W(x)-x=\frac{\bar{\sigma}_{1} W_{2}\left(W_{1}-x\right)+\bar{\sigma}_{2} W_{1}\left(W_{2}-x\right)+\bar{\sigma}_{1} \bar{\sigma}_{2} \overline{\bar{\sigma}}}{\bar{\sigma}_{2} W_{1}+\bar{\sigma}_{1} W_{2}} \geq 0
$$

since (i) holds. Similarly to show (ii) of $W$, we can use

$$
W(x)-\overline{\bar{\sigma}}=\overline{\bar{\sigma}} \frac{\left(W_{1}-\bar{\sigma}_{1}\right)\left(W_{2}-\bar{\sigma}_{2}\right)}{\bar{\sigma}_{2} W_{1}+\bar{\sigma}_{1} W_{2}} \geq 0
$$

since (ii) holds. To show (iii), we can use

$$
\begin{aligned}
& 3 x\left(x+\frac{\overline{\bar{\sigma}}^{2}}{3 x}-W\right)\left(\bar{\sigma}_{1} W_{2}+\bar{\sigma}_{2} W_{1}\right)=\left(3 x^{2}+\overline{\bar{\sigma}}^{2}\right)\left(\bar{\sigma}_{1} W_{2}+\bar{\sigma}_{2} W_{1}\right)-3 \overline{\bar{\sigma}}\left(W_{1} W_{2}+\bar{\sigma}_{1} \bar{\sigma}_{2}\right) x \\
& =\left(3 x^{2}+\overline{\bar{\sigma}}^{2}-3 x W_{1}\right) \bar{\sigma}_{1} W_{2}+\left(3 x^{2}+\overline{\bar{\sigma}}^{2}-3 x W_{2}\right) \bar{\sigma}_{2} W_{1}-3 \overline{\bar{\sigma}} \bar{\sigma}_{1} \bar{\sigma}_{2} x \\
& \geq\left(\overline{\bar{\sigma}}^{2}-\bar{\sigma}_{1}^{2}\right) \bar{\sigma}_{1} W_{2}+\left(\overline{\bar{\sigma}}^{2}-\bar{\sigma}_{2}^{2}\right) \bar{\sigma}_{2} W_{1}-3 \overline{\bar{\sigma}} \bar{\sigma}_{1} \bar{\sigma}_{2} x \\
& \geq\left(\overline{\bar{\sigma}}^{2}-\bar{\sigma}_{1}^{2}\right) \bar{\sigma}_{1} x+\left(\overline{\bar{\sigma}}^{2}-\bar{\sigma}_{2}^{2}\right) \bar{\sigma}_{2} x-3 \overline{\bar{\sigma}} \bar{\sigma}_{1} \bar{\sigma}_{2} x=0 .
\end{aligned}
$$


The property (iv) of $W$ follows since (iii) and (iv) assure that the $\dot{W}_{i}$ terms in (26) are nonnegative. Similary, (i) together with (v) assure that the $\dot{\bar{\sigma}}_{i}$ terms are non-negative. The property (v) and (vi) follow immediately from $\overline{\bar{\sigma}}=\sum_{i} \bar{\sigma}_{i}$.

The general case $(n>2)$ now follows by induction.

\section{TIME-VARYING TRANSFORMATIONS}

Time-varying transformations can be applied to improve the upper bound for time-varying linear systems. Here we will study the required conditions for these functions.

\section{A. Discrete Time}

Consider the observability Gramian inequality of the balanced realization

$$
A^{T}(t) \Sigma(t+1) A(t)+C^{T}(t) C(t) \leq \Sigma(t) .
$$

If $\Sigma(T+1)$ and $\Sigma(T)$ are given such that (27) holds, we can modify $\Sigma(T)$ by applying a PROI function: $W(\Sigma)$.

For instance we can use $W(\Sigma)=\Sigma+\alpha^{2}(T) \Sigma^{-1}(T)$ where $\alpha(T) \geq 0$. We can then continue in reverse time going from $t=T$ down to 0 . For each $t$ we have to assure that $\alpha(t) \geq \alpha(t+1)$; we may assume that $\alpha(T+1)=0$. If we let $Q(t)=\Sigma(t)+\alpha^{2}(t) \Sigma^{-1}(t)$ we have obtained a new observability Gramian that satisfies

$$
A^{T}(t) Q(t+1) A(t)+C^{T}(t) C(t) \leq Q(t), \quad t \in[0, T]
$$

With this new observability Gramian (still diagonal) we can perform a new balancing such that $\bar{\Sigma}^{2}(t)=\Sigma(t)\left(\Sigma(t)+\alpha^{2}(t) \Sigma^{-1}(t)\right)=\Sigma^{2}(t)+\alpha^{2}(t) I$.

In the general discrete-time case, the following lemma holds.

Lemma 7: Let $S$ be a time-varying PROI function. If $\Sigma(t)$ satisfies the observability Lyapunov inequality (15) and in addition either

$$
S(t, \Sigma(t)) \geq S(t+1, \Sigma(t)), \quad \text { or } \quad S(t, \Sigma(t+1)) \geq S(t+1, \Sigma(t+1))
$$

holds, then also $\bar{\Sigma}(t)=S(t, \Sigma(t))$ satisfies (15).

Similarly, let $R$ be a time-varying PROI function. If $\Sigma(t)$ satisfies the reachability Lyapunov inequality (16) and in addition either

$$
R(t+1, \Sigma(t)) \geq R(t, \Sigma(t)), \quad \text { or } \quad R(t+1, \Sigma(t+1)) \geq R(t, \Sigma(t+1))
$$


holds, then also $\bar{\Sigma}(t)=R(t, \Sigma(t))$ satisfies (16).

Proof: From Lemma 5 and (15) we have

$$
\begin{array}{r}
A^{T}(t) S(t, \Sigma(t+1)) A(t)+C^{T}(t) C(t) \leq S(t, \Sigma(t)), \text { and } \\
A^{T}(t) S(t+1, \Sigma(t+1)) A(t)+C^{T}(t) C(t) \leq S(t+1, \Sigma(t)) .
\end{array}
$$

Provided either $S(t, \Sigma(t)) \geq S(t+1, \Sigma(t))$ or $S(t, \Sigma(t+1)) \geq S(t, \Sigma(t+1))$ holds, we obtain

$$
A^{T}(t) S(t+1, \Sigma(t+1)) A(t)+C^{T}(t) C(t) \leq S(t, \Sigma(t)), \quad t \in[0, T] .
$$

The reachability part of the lemma can be shown similarly.

The time-varying PROI functions are monotonic (increasing or decreasing) functions. It is enough to ensure monotonicity at every $\sigma_{i} \in \Sigma$.

\section{B. Continuous Time}

For the general continuous-time case, the following lemma holds.

Lemma 8: Let $S$ be a time-varying PROI function. If $\Sigma(t)$ satisfies the observability Lyapunov inequality (17) and in addition

$$
\frac{\partial S}{\partial t}(t, \Sigma(t)) \leq 0
$$

holds, then also $\bar{\Sigma}=S(t, \Sigma)$ satisfies (17).

Similarly, let $R$ be a time-varying PROI function. If $\Sigma(t)$ satisfies the reachability Lyapunov inequality (18) and in addition

$$
\frac{\partial R}{\partial t}(t, \Sigma(t)) \geq 0
$$

holds, then also $\bar{\Sigma}=R(t, \Sigma)$ satisfies (18).

Proof: Applying a time-varying PROI function, $S$, on $\Sigma$ such that $Q(t)=S(t, \Sigma(t))$ yields

$$
S(t, \Sigma(t)) A(t)+A^{T}(t) S(t, \Sigma(t))+\frac{d S(t, \Sigma(t))}{d t}+C^{T}(t) C(t) \leq 0
$$

Using Lemma 5 yields

$$
\underbrace{S(t, \Sigma(t)) A(t)+A^{T}(t) S(t, \Sigma(t))+\frac{\partial S}{\partial \Sigma} \dot{\Sigma}(t)+C^{T}(t) C(t)}_{\leq 0 \text { since } S \text { is a PROI function }}+\frac{\partial S}{\partial t}(t, \Sigma(t)) \leq 0 .
$$

Since $\frac{\partial S}{\partial t}(t, \Sigma)$ is a diagonal matrix, it is enough to check each diagonal component. Thus, $S(t, \Sigma)$ satisfies the observability Lyapunov inequality if (28) holds.

Similarly, $R(t, \Sigma)$ satisfies the reachability Lyapunov inequality if $R$ is a PROI function satisfying (29). 


\section{Monotonic Functions}

A simple PROI function is $W_{2}(\sigma)=\sigma+\alpha^{2} / \sigma$. It is increasing for any $\sigma>0$ if we assume that $\dot{\alpha} \geq 0$.

A more general set of monotonic PROI functions can be described by

$$
W_{n}(\alpha, \sigma)=\alpha w_{n}\left(\frac{\sigma}{\alpha}\right)
$$

where

$$
w_{n}(x)= \begin{cases}n \frac{x^{n}+\left(\begin{array}{l}
n \\
2
\end{array}\right) x^{n-2}+\ldots+1}{n x^{n-1}+\left(\begin{array}{l}
n \\
3
\end{array}\right) x^{n-3}+\ldots+n x} & n \text { even, } \\
n \frac{x^{n}+\left(\begin{array}{l}
n \\
2
\end{array}\right) x^{n-2}+\ldots+n x}{n x^{n-1}+\left(\begin{array}{l}
n \\
3
\end{array}\right) x^{n-3}+\ldots+1} & n \text { odd. }\end{cases}
$$

The function $w_{n}(x)$ is increasing with respect to $x$ for $x \geq 1$.

We also have that $w_{n}(n x) / n \rightarrow \operatorname{coth}(1 / x)$ as $n \rightarrow \infty$ :

$$
w_{\infty}(x)=\operatorname{coth}\left(\frac{1}{x}\right)=x+\frac{1}{3 x+\frac{1}{5 x+\frac{1}{\ddots}}} .
$$

This yields $W_{\infty}(\alpha, x)=\alpha \operatorname{coth}(\alpha / x)$, which is monotonic for every fixed $x \geq 0$ if $\alpha$ is monotonic. This transformation can be applied to both discrete and continuous time systems.

\section{Monotonicity for Combined Functions}

We will now show how we can assure monotonicity at $\sigma=\sigma_{i} \in \Sigma=\left\{\sigma_{1}, \ldots, \sigma_{n}\right\}$, using a carefully designed PROI function. We will here consider the sets of removed Hankel singular values, $\Sigma_{N}$, and remaining singular value, $\Sigma_{R}$, differently.

We start by assuring monotonicity at $\sigma=\sigma_{i} \in \Sigma_{N}=\left\{\sigma_{r+1}, \ldots, \sigma_{n}\right\}$, using a PROI function defined by

$$
W(x)=\overline{\bar{\sigma}}\left(\bigoplus_{i=r+1}^{n} \frac{W_{i}(x)}{\bar{\sigma}_{i}}\right) \oplus\left(\bigoplus_{i=r+2}^{n} \frac{W_{i}(x)}{\bar{\sigma}_{i}}\right)
$$

where $\overline{\bar{\sigma}}=\bar{\sigma}_{r+1}+2 \sum_{i=r+2}^{n} \bar{\sigma}_{i}$ and $\bar{\sigma}_{i}=W_{i}\left(\sigma_{i}\right)$. Note that (34) defines a PROI function in which $\sigma_{r+2}, \ldots, \sigma_{n}$ appear twice and $\sigma_{r+1}$ only once. It is straightforward to show that

$$
\frac{\partial}{\partial t}\left(\overline{\bar{\sigma}}\left(\frac{W_{1}}{\bar{\sigma}_{1}}\right) \oplus\left(\frac{W_{1}}{\bar{\sigma}_{1}}\right) \oplus\left(\frac{W_{2}}{\bar{\sigma}_{2}}\right)\right)=2 \dot{\bar{\sigma}}_{1}+\dot{\bar{\sigma}}_{2}=\dot{\overline{\bar{\sigma}}}
$$


when evaluated at $\bar{\sigma}_{1}=W_{1}\left(\sigma_{1}\right)$. Thus, monotonicity is assured in all $\sigma_{r+2}, \ldots, \sigma_{n}$, since they are repeated (at least) twice if we assume that $\dot{\bar{\sigma}} \geq 0$.

Next, we verify that the PROI function is monotonic for any $x \geq \sigma_{r+1}$, which is the largest singular value in $\Sigma_{N}$.

Lemma 9 (Monotonicity): Let $W_{i}(x)=\alpha_{i} / \tanh \left(\alpha_{i} / x\right), \bar{\sigma}_{i}=W_{i}\left(\sigma_{i}\right)$ and $\overline{\bar{\sigma}}=\sum_{i=r+1}^{n} \bar{\sigma}_{i}$, where $\alpha_{i} \geq 0$. Then, the PROI function

$$
W(x)=\overline{\bar{\sigma}} \bigoplus_{i} \frac{W_{i}(x)}{\bar{\sigma}_{i}}
$$

is increasing, $\dot{W}(x) \geq 0$, for any fixed $x \geq \max _{i} \sigma_{i}=\sigma_{r+1}$, assuming $\dot{\bar{\sigma}}_{i} \geq 0$ and $\dot{W}_{i}(x) \geq 0$. Similarly, $W(x)$ is decreasing, $\dot{W}(x) \leq 0$, assuming $\dot{\bar{\sigma}}_{i} \leq 0$ and $\dot{W}_{i}(x) \leq 0$.

Proof: For any $x \geq \sigma_{r+1}$, it is easy to verify that $W_{i}(x)$ satisfy the properties in Lemma 6 . Consequently, $W(x)$ also do so.

In the discrete-time case we can for instance use

$$
\frac{W(t+1, x)}{\overline{\bar{\sigma}}(t+1)}=\left(\bigoplus_{i=r+1}^{n} \frac{W_{i}(t, x)}{\bar{\sigma}_{i}(t)}\right) \oplus\left(\bigoplus_{i=r+2}^{n} \frac{W_{i}(t+1, x)}{\bar{\sigma}_{i}(t+1)}\right)
$$

where

$$
\overline{\bar{\sigma}}(t+1)=\bar{\sigma}_{r+1}(t)+\sum_{i=r+2}^{n}\left(\bar{\sigma}_{i}(t)+\bar{\sigma}_{i}(t+1)\right) .
$$

Here, $\bar{\sigma}_{r+2}, \ldots, \bar{\sigma}_{n}$ appear twice, ones for $t$ and another time for $t+1$, while $\bar{\sigma}_{r+1}$ only appears once.

\section{SHUtTLE APPROACHES}

We will now use time-varying transformations to modify the Hankel singular values so that the smallest one, $\sigma_{n}(t)$, becomes constant over time. After removing the corresponding state, we can proceed recursively until the reduced model is of order $r$.

In this section we will study various approaches to find upper bounds of the model error. Three variants will be considered

- Each pass is followed by rebalancing;

- Two independent passes followed by rebalancing;

- Coordinated passes. 


\section{A. Each Pass Followed by Rebalancing}

In the first approach we will move forward and backward, just like passing a shuttle through the shed in a loom. In each cycle one Hankel singular value (possibly repeated) is removed.

This is done by first modifying the reachability Gramian, $P$, in forward time, starting at $t=0$. By choosing a suitable time-varying transformation we can force the smallest singular value (after another balancing step) to become an increasing function in time. In the final step, the observability Gramian, $Q$, is modified in backward time so that the smallest singular value becomes constant after yet another rebalancing. The following discussion covers the discrete-time case, while the continuous-time case can be handled analogously.

We start by modifying the reachability Gramian, $P$. Let $P(t)=W(\Sigma)=\Sigma(t)+\alpha_{p}^{2}(t) \Sigma^{-1}$ and $Q(t)=\Sigma(t)$. The transformation used here, $W$, is obviously increasing for any $\sigma$, if $\alpha_{p}$ is increasing. After rebalancing, the smallest modified singular value, $\bar{\sigma}_{n}$, becomes

$$
\bar{\sigma}_{n}^{2}(t)=p_{n}(t) q_{n}(t)=\left(\sigma_{n}(t)+\frac{\alpha_{p}^{2}(t)}{\left.\sigma_{n}(t)\right)}\right) \sigma_{n}(t)=\sigma_{n}^{2}(t)+\alpha_{p}^{2}(t) .
$$

We start by defining $\alpha_{p}(0)=0$ and consequently $\bar{\sigma}_{n}(0)=\sigma_{n}(0)$. We now work our way forward in time until $t=T+1$. In each step we have to assure that $\alpha_{p}(t+1) \geq \alpha_{p}(t)$ and $\bar{\sigma}_{n}(t+1) \geq \bar{\sigma}_{n}(t)$ in order to respect the monotonicity condition. We want to find the smallest $\alpha_{p}(t)$ and $\bar{\sigma}_{n}(t)$ that satisfy these conditions.

The rule to solve this problem is the following: if $\sigma_{n}(t+1) \leq \sigma_{n}(t)$ let $\bar{\sigma}_{n}(t+1)=\bar{\sigma}_{n}(t)$ and $\alpha_{p}^{2}(t+1)=\bar{\sigma}_{n}^{2}(t)-\sigma_{n}^{2}(t+1)$, otherwise keep $\alpha_{p}(t+1)=\alpha_{p}(t)$ and $\bar{\sigma}_{n}^{2}(t+1)=\sigma_{n}^{2}(t+1)+\alpha_{p}^{2}(t+1)$. We can also express this as $\alpha_{p}^{2}(t+1)=\alpha_{p}^{2}(t)+\max \left\{0, \sigma_{n}^{2}(t)-\sigma_{n}^{2}(t+1)\right\}$, which yields

$$
\bar{\sigma}_{n}^{2}(t+1)=\bar{\sigma}_{n}^{2}(t)+\max \left\{0, \sigma_{n}^{2}(t+1)-\sigma_{n}^{2}(t)\right\} .
$$

This means that in intervals where $\sigma_{n}$ is increasing, $\alpha_{p}$ becomes constant, and in intervals where $\sigma_{n}$ is decreasing we let $\alpha_{p}^{2}(t)=\bar{\sigma}_{n}^{2}\left(t_{m}(t)\right)-\sigma_{n}^{2}(t)$ where $t_{m}(t)$ denotes the first maximum of $\sigma_{n}(t)$ after $t$. We can thus show that

$$
\bar{\sigma}_{n}^{2}(T+1)=\sigma_{n}^{2}(0)+\sum_{t=0}^{T} \max \left\{0, \sigma_{n}^{2}(t+1)-\sigma_{n}^{2}(t)\right\} .
$$

Let $\sigma_{n}$ have $M$ local maxima, $\sigma_{n}\left(t_{\max , i}\right)$, in the interval $[0, T+1]$. Then $\sigma_{n}$ will have $M-1$ local minima, $\sigma_{n}\left(t_{\min , i}\right)$, in between $\sigma_{n}\left(t_{\max , 1}\right)$ and $\sigma_{n}\left(t_{\max , M}\right)$, so that

$$
0 \leq t_{\max , 1}<t_{\min , 1}<\ldots<t_{\min , M-1}<t_{\max , M} \leq T+1 .
$$


and define

$$
\mathcal{S}_{2}^{2}\left(\sigma_{n}\right)=\sigma_{n}^{2}\left(t_{\max , M}\right)+\sum_{i=1}^{M-1}\left(\sigma_{n}^{2}\left(t_{\max , i}\right)-\sigma_{n}^{2}\left(t_{\min , i}\right)\right) .
$$

After this processing, we have modified the smallest singular value to become an increasing function with $\bar{\sigma}_{n}^{2}(T+1)=\mathcal{S}_{2}^{2}\left(\sigma_{n}\right)$ and $\bar{\sigma}(0)=\sigma(0)$.

After rebalancing, the procedure is repeated in backward time starting at $t=T+1$ and modifying the observability Gramian, $Q$, so that $\overline{\bar{\sigma}}_{n}(t)=\overline{\bar{\sigma}}_{n}$ becomes constant over the entire interval $t \in[0, T+1]$. After a second rebalancing all singular values are modified as

$$
\overline{\bar{\sigma}}_{i}^{2}(t)=\bar{\sigma}_{n}^{2}+\alpha_{q}^{2}(t)=\sigma_{i}^{2}(t)+\alpha_{p}^{2}(t)+\alpha_{q}^{2}(t)=\overline{\bar{\sigma}}_{n}^{2}+\sigma_{i}^{2}(t)-\sigma_{n}^{2}(t),
$$

where

$$
\overline{\bar{\sigma}}_{n}^{2}=\bar{\sigma}_{n}^{2}(T+1)+\sum_{t=0}^{T} \max \{0, \underbrace{\bar{\sigma}_{n}^{2}(t)-\bar{\sigma}_{n}^{2}(t+1)}_{\leq 0}\}=\mathcal{S}_{2}^{2}\left(\sigma_{n}\right) .
$$

Note that $\mathcal{S}_{2}$ is symmetric with respect to time: it applies both for backward and forward passes.

Since the modified smallest Hankel singular value is constant over time, it can be removed with an error bounded defined by

$$
\left\|G-\hat{G}_{n-1}\right\| \leq \overline{\bar{\sigma}}_{n}=\mathcal{S}_{2}\left(\sigma_{n}\right) .
$$

By using balanced truncation (13) in all but the last step, we obtain the following upper bound,

Theorem 1: Let $G$ be an LPV system. Then there exists a reduced order LPV system, $\hat{G}_{r}$, of order $r$ such that

$$
\left\|G-\hat{G}_{r}\right\| \leq \mathcal{S}_{2}\left(\sigma_{r+1}\right)+2 \sum_{k=r+2}^{n} \mathcal{S}_{2}\left(\sigma_{k}\right) .
$$

where $\mathcal{S}_{2}$ is defined in (40).

Proof: Remove $\sigma_{n}, \sigma_{n-1}, \ldots, \sigma_{r+2}$ one by one using balanced truncation. The model error in each step is then bounded by $\mathcal{S}_{2}\left(\sigma_{i}\right)$. The remaining singular values are not affected. Finally, remove $\sigma_{r+1}$ using (11) or (12).

These bounds can be compared with the upper bound given in [14]:

$$
\left\|G-\hat{G}_{r}\right\| \leq 2 \sum_{k=r+1}^{n} \mathcal{S}_{\mathcal{T}}\left(\sigma_{k}\right), \quad \text { where } \quad \mathcal{S}_{\mathcal{T}}\left(\sigma_{k}\right)=\sigma_{k}\left(t_{\max , M}\right) \prod_{i=1}^{M-1} \frac{\sigma_{k}\left(t_{\max , i}\right)}{\sigma_{k}\left(t_{\min , i}\right)}
$$




\section{B. Independent Passes}

We will start to improve the bound given in (41) by performing two independent passes with transformations defined by

$$
W(x)=\alpha \operatorname{coth}(\alpha / x)
$$

After these two passes we modify the Gramians once more in order to obtain a constant modified singular value after the rebalancing.

We have to respect the monotonicity requirements so that the transformation, $S$, that modifies the observability Gramians becomes increasing and the one modifying the reachability Gramian, $R$, is decreasing. After we have achieved monotonic observability and reachability Gramians we can finally modify them to become constant after the concluding rebalancing.

We start by looking at the PROI function modifying the observability Gramian. The timevarying transformation must be increasing both at the singular value to be removed and any other singular values above this removed one. Using the transformation defined in (44) we can assure this by having an increasing $\alpha$ with respect to time, such that $\dot{\alpha} \geq 0$, which means that $S(t, \sigma)$ is increasing for every fixed $\sigma$. At the same time we need to assure that $\dot{\bar{\sigma}} \geq 0$, where $\bar{\sigma}=S\left(t, \sigma_{n}\right)$.

We can achieve this by the following rules. When $\sigma_{n}$ is increasing we keep $\alpha$ constant and when $\sigma_{n}$ is decreasing we increase $\alpha$ so that

$$
\bar{\sigma}_{n}(t)=S\left(t, \sigma_{n}(t)\right)=\frac{\alpha(t)}{\tanh \left(\frac{\alpha(t)}{\sigma_{n}(t)}\right)}=\sigma_{n}(t) f\left(\frac{\alpha(t)}{\sigma_{n}(t)}\right),
$$

becomes constant. Here $f$ denotes $f(x)=x \operatorname{coth}(x)$. Thus, assuming that the minima and maxima of $\sigma_{n}$ occurs as in (39) and using $\beta(t)=\alpha(t) / \sigma_{n}(t)$, we can express this as

$$
\begin{aligned}
\beta_{n}\left(t_{\max , 1}\right) & =1, \\
\beta_{n}\left(t_{\max , i+1}\right) & =f\left(\frac{\sigma_{n}\left(t_{\min , i}\right)}{\sigma_{n}\left(t_{\max , i+1}\right)} f^{-1}\left(\frac{\left.\sigma_{n}\left(t_{\max , i}\right)\right)}{\sigma_{n}\left(t_{\min , i}\right)} \beta_{n}\left(t_{\max , i}\right)\right)\right),
\end{aligned}
$$

where $f^{-1}$ denotes the inverse function of $f$. If $\sigma_{n}\left(t_{\min , i}\right)=0$ we can use

$$
\beta_{n}\left(t_{\max , i+1}\right)=f\left(\frac{\sigma_{n}\left(t_{\max , i}\right)}{\sigma_{n}\left(t_{\max , i+1}\right)} \beta_{n}\left(t_{\max , i}\right)\right) .
$$

Next, define

$$
\mathcal{S}_{C+}\left(\sigma_{n}\right)=\bar{\sigma}_{n}(T+1)=\beta_{n}\left(t_{\max , M}\right) \sigma_{n}\left(t_{\max , M}\right),
$$


where $\beta_{n}$ satisfies (45). Note that $\mathcal{S}_{C+}$ is expressed in terms of the local maxima and minima of $\sigma_{n}$, in a similar way as $\mathcal{S}_{2}$. Specifically, we can determine an upper bound for $\mathcal{S}_{C+}$ in terms of the number of local maxima, $M$, and the global maximum, $\sigma_{n, \max }$ :

$$
\beta_{i+1}=f\left(\beta_{i}\right)=\frac{\beta_{i}}{\tanh \beta_{i}}, \quad i=1, \ldots, M-1
$$

where $\beta_{1}=1$. This yields $\mathcal{S}_{C+}\left(\sigma_{n}\right) \leq \beta_{M} \sigma_{n \text {,max }}$. The upper error bound has been computed using the assumption that every local minimum is 0 and every local maximum is $\sigma_{\max }$. The function $\mathcal{S}_{C+}$ increases rather gracefully (roughly logarithmically) as a function of the number of maxima, $M$. The function $\mathcal{S}_{C-}$ is defined similarly for the modified reachability Gramian. Note that $\mathcal{S}_{C-}$ is not identical to $\mathcal{S}_{C+}$, even if their upper bounds as a function of number of minima are the same.

Finally, $S\left(t, \sigma_{n}(t)\right)$ and $R\left(t, \sigma_{n}(t)\right)$ are modified so that their product becomes constant over time. A simple, but not always optimal, way to achieve this is as follows. Since, $R\left(t, \sigma_{n}(t)\right)$ is decreasing, we modify $S\left(t, \sigma_{n}(t)\right)$ by applying another increasing transformation, $S_{2}$, such that $S_{2}\left(t, S\left(t, \sigma_{n}(t)\right)\right)=\gamma S\left(t, \sigma_{n}(t)\right) / R\left(t, \sigma_{n}(t)\right)$, where

$$
\gamma^{2}=\max _{t} S\left(t, \sigma_{n}(t)\right) \times \max _{t} R\left(t, \sigma_{n}(t)\right)=\mathcal{S}_{C+}\left(\sigma_{n}\right) \mathcal{S}_{C-}\left(\sigma_{n}\right)
$$

Similarly, $R\left(t, \sigma_{n}(t)\right)$ is modified by another decreasing transformation, $R_{2}$, such that $R_{2}\left(t, R\left(t, \sigma_{n}(t)\right)\right)=$ $\gamma R\left(t, \sigma_{n}(t)\right) / S\left(t, \sigma_{n}(t)\right)$. After rebalancing, this yields

$$
\bar{\sigma}_{n}(t)=\sqrt{R_{2}\left(t, R\left(t, \sigma_{n}(t)\right)\right) S_{2}\left(t, S\left(t, \sigma_{n}(t)\right)\right)}=\gamma .
$$

Thus, the model error using this method with independent passes is bounded by

$$
\left\|G_{n}-\hat{G}_{n-1}\right\| \leq \mathcal{S}_{C}\left(\sigma_{n}\right)=\sqrt{\mathcal{S}_{C+}\left(\sigma_{n}\right) \mathcal{S}_{C-}\left(\sigma_{n}\right)} .
$$

\section{Removing Several Singular Values}

In order to remove several singular values at a time, we first transform each singular value, $\sigma_{i} \in \Sigma_{N}$, using time-varying $S_{i}$ and $R_{i}$. Finally, we combine these transformations into $S$ and $R$, using (34) or (37), which results in $n-r$ equal and monotonic singular values. Finally, we transform these so that they become constant after the final rebalancing step. This yields the following upper bound

$$
\left\|G-\hat{G}_{r}\right\| \leq \sqrt{\mathcal{S}_{C+}\left(\sigma_{r+1}\right)+2 \sum_{i=r+2}^{n} \mathcal{S}_{C+}\left(\sigma_{i}\right)} \times \sqrt{\mathcal{S}_{C-}\left(\sigma_{r+1}\right)+2 \sum_{i=r+2}^{n} \mathcal{S}_{C-}\left(\sigma_{i}\right)} .
$$


A similar, simpler, and marginally better, bound can be obtained by using balanced truncation for the last $n-r-1$ singular values and finally using (47) in the last step when removing $\sigma_{r+1}$. Thus, we have shown the following bound.

Theorem 2: Let $G$ be an LPV system. Then there exists a reduced order LPV system, $\hat{G}_{r}$, of order $r$ such that

$$
\left\|G-\hat{G}_{r}\right\| \leq \mathcal{S}_{C}\left(\sigma_{r+1}\right)+2 \sum_{i=r+2}^{n} \mathcal{S}_{C}\left(\sigma_{i}\right) .
$$

where $\mathcal{S}_{C}$ is defined in (47) and (46).

\section{Coordinated Passes}

The bound in (49), even for the case $r=n-1$, is not always the best. The reason for this is that the singular values are modified in two independent passes: the first in forward and the second in backward time. The resulting modified Gramians are in turn modified so that their Hankel singular values become constant over time by another pair of transformations.

We will see how combining these transformations into one pair of transformations (instead of two) can improve the bounds. We will call this the method with coordinated passes, which can be described as follows. Search for time-varying transformations functions, $R$ and $S$, such that $\bar{\sigma}_{n}=\sqrt{R\left(t, \sigma_{n}(t)\right) S\left(t, \sigma_{n}(t)\right)}$ becomes constant and minimal. As before $S$ is the transformation of the observability Gramian, $Q(t)=S(t, \Sigma(t))$, and $R$ modifies the reachability Gramian, $P(t)=R(t, \Sigma(t))$. We have to assure that $S\left(t, \sigma_{i}\right) \geq S_{t+1}\left(\sigma_{i}\right)$ and $R\left(t, \sigma_{i}\right) \leq R\left(t+1, \sigma_{i}\right)$ for $i \in[1, n]$.

The drawback with coordinated passes is that it makes the computation more involved and complex, while the reduction in the bound is typically $10-15 \%$ compared to using independent passes. We will now illustrate this method using a few different transformation functions. Let us consider a discrete-time (or continuous-time) system over a time horizon with two maxima of $\sigma_{n}$ at $t=0$ and $t=T+1$, both with $\sigma_{n}(t)=1$. In between these points there is a minimum, $\sigma_{\min }$, at $t_{\min }$. According to (41) or (47) we can obtain $\mathcal{S}_{2}\left(\sigma_{n}\right)$ or $\mathcal{S}_{C}\left(\sigma_{n}\right)$ as upper bounds.

We will now try to improve this bound. First, let

$$
\begin{aligned}
& R(t, \sigma)=\sigma+\alpha_{R}^{2}(t) / \sigma=\alpha_{R}(t) W_{2}\left(\sigma / \alpha_{R}(t)\right), \\
& S(t, \sigma)=\sigma+\alpha_{S}^{2}(t) / \sigma=\alpha_{S}(t) W_{2}\left(\sigma / \alpha_{S}(t)\right) .
\end{aligned}
$$


TABLE I

IMPROVED BOUNDS WITH ONE MINIMUM USING A SECOND-ORDER RATIONAL FUNCTION, $W(\sigma)=\sigma+\alpha^{2} / \sigma$. IN ORDER TO ASSURE MONOTONICITY WE ASSUME THAT $\sigma_{\mathrm{MIN}} \geq 1 / \sqrt{3}$.

\begin{tabular}{|c|c|c|c|c|c|c|}
\hline$t$ & $\sigma_{n}(t)$ & $\alpha_{R}$ & $R(t, \sigma)$ & $\alpha_{S}$ & $S(t, \sigma)$ & $\bar{\sigma}_{n}^{2}$ \\
\hline 0 & 1 & 1 & $\sigma$ & $\alpha_{1}$ & $\sigma+\frac{\alpha_{1}^{2}}{\sigma}$ & $1+\alpha_{1}^{2}$ \\
$t_{\min }$ & $\sigma_{\min }$ & $\alpha_{1}$ & $\sigma+\frac{\alpha_{1}^{2}}{\sigma}$ & $\alpha_{1}$ & $\sigma+\frac{\alpha_{1}^{2}}{\sigma}$ & $\left(\sigma_{\min }+\frac{\alpha_{1}^{2}}{\sigma_{\min }}\right)^{2}$ \\
$T+1$ & 1 & $\alpha_{1}$ & $\sigma+\frac{\alpha_{1}^{2}}{\sigma}$ & 1 & $\sigma$ & $1+\alpha_{1}^{2}$ \\
\hline
\end{tabular}

TABLE II

IMPROVED BOUNDS WITH ONE MINIMUM USING AN INFINITE-ORDER TRANSFORMATION, $W_{\infty}(\sigma)=\alpha \operatorname{coth}(\alpha / \sigma)$. IN ORDER to ACHIEVE A CONSTANT $\bar{\sigma}$, WE LET $\alpha_{1} \operatorname{coth}^{2}\left(\alpha_{1} / \sigma_{\text {Min }}\right)=\operatorname{coth} \alpha_{1}$. SPECIFICALLY WHEN $\sigma_{\text {MIN }}=0$, WE GET $\alpha_{1}=\operatorname{coth} \alpha_{1}$, WHICH YIELDS $\bar{\sigma}_{n}=\alpha_{1}=1.19968$.

\begin{tabular}{|c|c|c|c|c|}
\hline$t$ & $\sigma_{n}(t)$ & $R(t, \sigma)$ & $S(t, \sigma)$ & $\bar{\sigma}_{n}^{2}$ \\
\hline 0 & 1 & $\sigma$ & $\alpha_{1} W_{\infty}\left(\frac{\sigma}{\alpha_{1}}\right)$ & $\alpha_{1} \operatorname{coth} \alpha_{1}$ \\
$t_{\min }$ & $\sigma_{\min }$ & $\alpha_{1} W_{\infty}\left(\frac{\sigma}{\alpha_{1}}\right)$ & $\alpha_{1} W_{\infty}\left(\frac{\sigma}{\alpha_{1}}\right)$ & $\alpha_{1}^{2} \operatorname{coth}\left(\frac{\alpha_{1}}{\sigma_{\min }}\right)$ \\
$T+1$ & 1 & $\alpha_{1} W_{\infty}\left(\frac{\sigma}{\alpha_{1}}\right)$ & $\sigma$ & $\alpha_{1} \operatorname{coth} \alpha_{1}$ \\
\hline
\end{tabular}

If $\sigma \geq \alpha$, these transformations have the property that they are monotonic in both $\alpha$ and $\sigma$. Specifically, $\alpha W_{n}(\sigma / \alpha)$ is an increasing function in both $\alpha$ and $\sigma$.

We now need to find monotonic $\alpha_{R}$ and $\alpha_{S}$ such that $\bar{\sigma}_{n}$ becomes constant, see the last column of Table I. Thus $\bar{\sigma}_{n}^{2}=\left(\sigma_{\min }+\alpha_{1}^{2} / \sigma_{\min }\right)^{2}=1+\alpha_{1}^{2}$, which has the solution $\alpha_{1}^{2}=$ $\frac{1}{2} \sigma_{\min }\left(\sqrt{4-3 \sigma_{\min }^{2}}-\sigma_{\min }\right)$. Further we must assure that $\sigma_{\min } \geq \alpha_{1}$ in order to stay in the monotonic region of the transformation, which implies $\sigma_{\min } \geq 1 / \sqrt{3}$. When $\sigma_{\min }=1 / \sqrt{3}$ we get $\bar{\sigma}_{n}=\sqrt{\frac{4}{3}}$ compared to $\sqrt{2-\sigma_{\min }^{2}}=\sqrt{\frac{5}{3}}$ obtained from (41).

We can now go on with higher-order PROI functions of the type $\alpha W_{n}(\sigma / \alpha)$. By increasing the order $n$ of the rational function we can cover down to any minimum $\sigma_{\min }>0$. In the limit, see Table II, we get $\bar{\sigma}_{n}=1.19968$ for $\sigma_{\min }=0$, with $W_{\infty}(x)=\operatorname{coth}(1 / x)\left(\bar{\sigma}_{n}=\alpha_{1}\right.$ is the solution to $\operatorname{coth} \alpha_{1}=\alpha_{1}$ ). The different bounds for this case are summarized in Figure 1.

The upper error bound can be expressed as a function of the maximum of $\sigma_{n}$ and the number of minima, $M$, in the interval between the first and the last maxima. Using carefully designed 


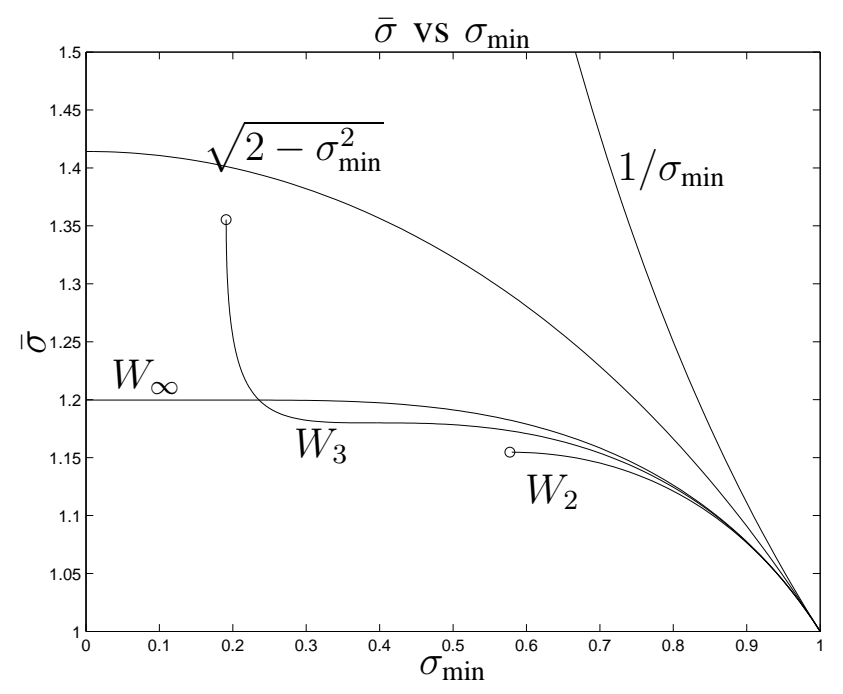

Fig. 1. Comparison of upper error bounds for a system with a singular value, $\sigma_{n}$, that is 1 at both end points and has one minimum of $\sigma_{\min }$ in the middle. The bound labelled $1 / \sigma_{\min }$ is from [14], while the bound labelled $\sqrt{2-\sigma_{\min }^{2}}$ is from (41). The bounds labelled $W_{2}$ and $W_{3}$ are derived using the second and third order rational functions, respectively, while $W_{\infty}$ uses $\alpha \operatorname{coth}(\alpha / x)$ to transform the Hankel singular values. Note that $W_{\infty}$ does not always provide the best bound, but is flexible since it can handle $\sigma_{\min }$ down to any $\epsilon>0$, which the others cannot. Also note that all bounds are asymptotically similar close to $\sigma_{\min }=1$.

transformation functions we can derive a bound that grows roughly logarithmically with respect to $M$, see Table III. As a comparison, the bound in (42) gives an upper bound that grows as the square root of $M: \sigma_{\max } \sqrt{1+M}$. The bound in (43) grows exponentially or linearly (by splitting the interval into smaller parts) as the number of minima.

Several minima can be analyzed using the same technique as for independent passes, that is using balanced truncation and optimal model reduction. Then a bound similar to (49) can be obtained. We just need to replace $\mathcal{S}_{C}$ with its improved version using coordinated passes.

\section{E. An Example}

As an example to illustrate this technique we take the diesel exhaust catalyst model from [14], where it is reduced from 24th to first order using balanced truncation. The corresponding uppder bound of the model error is $48 \times 10^{-3}$. The simulated model error is $7.2 \times 10^{-3}$, so the gap between the upper bound and the actual error is quite large.

The two largest Hankel singular values, $\sigma_{1}$ and $\sigma_{2}$, dominate over the the remaining singular values, so we can neglect the effects of all but the these two. The second largest singular 
TABLE III

THE UPPER MODEL ERROR BOUND, $\bar{\sigma}_{n} / \sigma_{\text {MAX }}$, FOR REMOVING ONE STATE, AS A FUNCTION OF THE NUMBER OF MINIMA, $M$, IN $\sigma_{n}$. THE COLUMN LABELED coord APPLIES TO A COORDINATED PASS WHILE INDEPENDENT PASSES IS LABELED indep

\begin{tabular}{|c|c|c|c|}
\hline$M$ & coord & indep & $\sqrt{n+1}$ \\
\hline 0 & 1 & 1 & 1 \\
1 & 1.1997 & 1.3130 & 1.4142 \\
2 & 1.3533 & 1.5179 & 1.7321 \\
3 & 1.4769 & 1.6711 & 2 \\
4 & 1.5800 & 1.7936 & 2.2370 \\
5 & 1.6685 & 1.8957 & 2.4495 \\
10 & 1.9844 & 2.2447 & \\
100 & 3.2707 & 3.5573 & 10.0499 \\
$10^{4}$ & 5.8991 & 6.1603 & \\
$10^{6}$ & 8.3985 & 8.6476 & \\
\hline
\end{tabular}

value has a maximum of about $6 \times 10^{-3}$ and has three minima in between the maxima. Thus, using the method with coordinated passes and balanced truncation gives an upper bound of $2 \times 1.48 \times 6 \times 10^{-3}=18 \times 10^{-3}$. Using, (11) allows us to halve the error bound down to $9 \times 10^{-3}$, only $25 \%$ higher than the simulated model error.

\section{CONSTANT UPPER BOUNDS FOR INFINITE TIME HoRIZONS}

So far we arrived at an ever increasing upper bound, $\gamma$, as a function of the time horizon or number of minima. Even if it is slowly increasing, we would also like to find upper bounds that do not depend on the length of the time interval. This is specifically relevant for periodic systems. To achieve this, we have to let go of the assumption that the time-varying transformation is monotonic for all $\sigma$ above the largest removed singular value (see Lemma 9). Instead we have to assure monotonicity only for the actual remaining singular values, $\Sigma_{R}=\left\{\sigma_{1}, \ldots, \sigma_{r}\right\}$ in addition to the removed singular values $\Sigma_{N}=\left\{\sigma_{r+1}, \ldots, \sigma_{n}\right\}$. This has the consequence that we need more specific knowledge about the remaining singular values in terms of their magnitude as a function of time. 


\section{A. Bounds for Zeroth-Order Models}

We can use the results in lemmas 7 and 8 in order to give an upper bound for reducing an $n$ th-order LTV system to zeroth order (or any order).

Theorem 3: Let $G$ be an LPV system. Then there exists a reduced order LPV system, $\hat{G}_{r}$, system such that $\left\|G-\hat{G}_{r}\right\| \leq \sup _{t} \bar{\sigma}(t)$, where

$$
\bar{\sigma}(t)= \begin{cases}2 \sum_{i=1}^{n} \sigma_{i}(t), & \text { continuous-time case } \\ \sum_{i=1}^{n}\left(\sigma_{i}(t)+\sigma_{i}(t+1)\right), & \text { discrete-time case. }\end{cases}
$$

Proof: In the discrete-time case we can use

$$
S=R=W
$$

where $W$ is a time-varying transformation defined by

$$
\left\{\begin{array}{l}
W(t, \Sigma(t))=W(t, \Sigma(t+1))=\gamma, \text { and } \\
\bar{\sigma}(t)=\sum_{i}\left(\sigma_{i}(t)+\sigma_{i}(t+1)\right), \\
\gamma=\sup _{t} \bar{\sigma}(t) .
\end{array}\right.
$$

For instance we can use

$$
\frac{W(t, x)}{\gamma}=\frac{x}{\gamma-\bar{\sigma}_{i}(t)} \oplus\left(\bigoplus_{i}^{n} \frac{x}{\sigma_{i}(t)}\right) \oplus\left(\bigoplus_{i}^{n} \frac{x}{\sigma_{i}(t+1)}\right)
$$

which yields

$$
W(t, \Sigma(t+1))=W(t+1, \Sigma(t+1))
$$

so that the conditions in Lemma 7 hold. The continuous-time case proof is similar.

As a matter of fact, reducing an LTV model to zeroth order is a convex problem, since we can use the fact that the product of the observability and reachability Gramians is a unit matrix times a constant: $P Q=\gamma^{2} I$, see [9], [22]. This yields

$$
\left\{\begin{array}{l}
A(t) P(t) A^{T}(t)+B(t) B^{T}(t) \leq P(t+1), \\
{\left[\begin{array}{l}
A(t) \\
C(t)
\end{array}\right] P(t)\left[\begin{array}{l}
A(t) \\
C(t)
\end{array}\right]^{T} \leq\left[\begin{array}{cc}
P(t+1) & 0 \\
0 & \gamma^{2} I
\end{array}\right],}
\end{array}\right.
$$

where the second LMI has been obtained from the observability Lyapunov inequality (2) using Schur complements. 
These inequalities, together with the condition $P(t)>0$, can be used to compute the minimum $\gamma$ as an LMI program. In continuous time the problem becomes infinite-dimensional, but still convex.

\section{B. Discrete-Time Bounds}

Bounds for reducing an $n$ th-order LTV system to order $r>0$ is more involved and will be elaborated in the sequel. We will here start with using the condition, $W(t, \Sigma(t+1))=$ $W(t+1, \Sigma(t+1))$ from Lemma 7 , which can be expressed as

$$
W\left(t, \sigma_{i}(t+1)\right)=W\left(t+1, \sigma_{i}(t+1)\right) .
$$

For the Hankel singular values that are removed, $\Sigma_{N}=\left\{\sigma_{r+1}, \ldots, \sigma_{n}\right\}$, we assume that

$$
W\left(t, \sigma_{i}(t+1)\right)=W\left(t+1, \sigma_{i}(t+1)\right)=\bar{\sigma}, \quad \sigma_{i} \in \Sigma_{N}
$$

for some constant $\bar{\sigma}$. The transformation, $W$, evaluated at the remaining singular values are instead fixed to a time-invariant template function, $\tau_{\bar{\sigma}}$. For $\Sigma_{R}=\left\{\sigma_{1}, \ldots, \sigma_{r}\right\}$ assume that

$$
W\left(t, \sigma_{k}(t+1)\right)=W\left(t+1, \sigma_{k}(t+1)\right)=f_{\bar{\sigma}}\left(\sigma_{k}(t+1)\right), \quad \sigma_{k} \in \Sigma_{R}
$$

The template function, $\tau_{\bar{\sigma}}$, can be chosen freely, but

$$
\tau_{\bar{\sigma}}(x)=\bar{\sigma} \operatorname{coth} \frac{\bar{\sigma}}{x}
$$

is used in this paper, since it has some nice properties:

- it is a PROI function, since $\tau_{\bar{\sigma}}(x)-x$ is positive real;

- if $\bar{\sigma}$ satisfies the interpolation condition, then also any value greater than $\bar{\sigma}$ will do so.

- the upper bound can be computed as a minimum of $T+1$ values, each if which only depends on $\Sigma(t)$ at that very instant, $t$.

Shifting the argument $t$ yields

$$
W\left(t, \sigma_{i}\right)= \begin{cases}\tau_{\bar{\sigma}}\left(\sigma_{i}\right), & \text { for } \sigma_{i} \in \Sigma_{R}(t) \cup \Sigma_{R}(t+1), \\ \bar{\sigma}, & \text { for } \sigma_{i} \in \Sigma_{N}(t) \cup \Sigma_{N}(t+1) .\end{cases}
$$

We can regard this as an interpolation problem using positive-real, odd functions. Figure 2 illustrates this for an example where $n=2$ and $r=1$ with $\sigma_{1}(t)=0.8, \sigma_{1}(t+1)=0.7$, $\sigma_{2}(t)=0.6$ and $\sigma_{2}(t+1)=0.5$. 


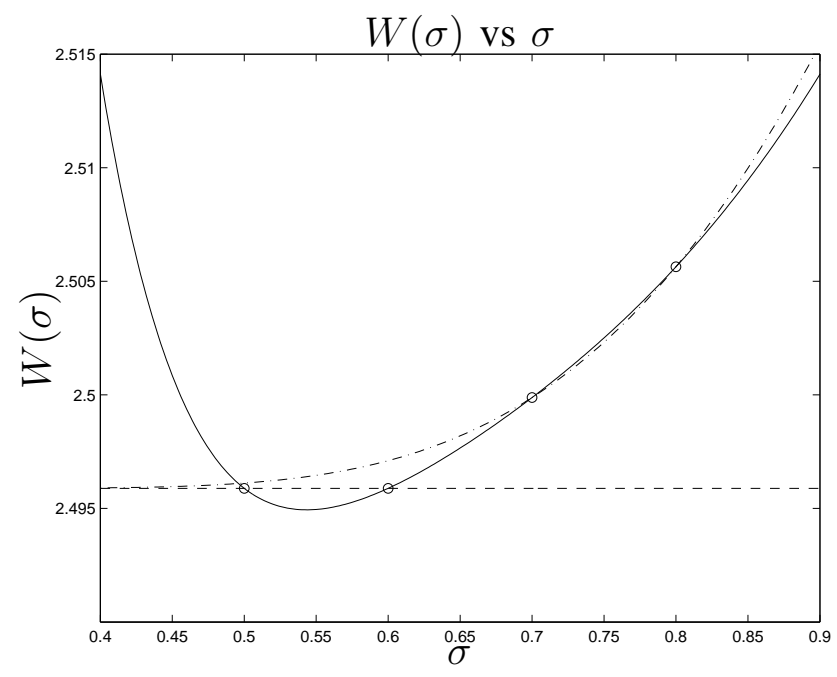

Fig. 2. Interpolation problem for a discrete-time, second-order system to be reduced to first order. Here $\sigma_{2}(t)=0.8, \sigma_{2}(t+1)=$ $0.7, \sigma_{1}(t)=0.6$ and $\sigma_{1}(t+1)=0.5$. The PROI function (solid line) crosses the template function (dash-dotted) line at $\sigma=0.7$ and 0.8 , while it goes through the line $W=\bar{\sigma}$ (dashed) at $\sigma=0.5$ and 0.6 . This yields the minimum $\bar{\sigma}=2.4959$.

Note the specific choice of template function will affect the bound. The template function in (56) is not the only choice. We can even consider to use time-varying template functions that respect certain properties. However, even if this method, with a fixed template function, does not obtain the best upper bound, it allows us to treat each time instant independently, by minimizing $\bar{\sigma}$ with respect to (57). This can be done efficiently using the Nevanlinna-Pick condition, for instance by searching for the minimum by a bisection algorithm.

An alternative to the template function approach is to formulate a set of LMIs with $T+1$ Nevanlinna-Pick conditions, one for each $W(t, x)$. The LMIs are parametrized with $\bar{\sigma}$ and the $r(T+1)$ values corresponding to (53) for $i=1, \ldots, r$. This works for small problems with short time horizons, $T$, and a few number of states, $n$. For large problems the number of variables in the LMIs will increase, which extends the solution time. In addition, numerical problems are more likely to occur. 


\section{Continuous-time Bounds}

In the continuous-time case we can proceed similarly, assuming $S=R=W$ such that $\frac{\partial W}{\partial t}\left(t, \sigma_{i}(t)\right)=0$

$$
W\left(t, \sigma_{i}\right)= \begin{cases}\tau_{\bar{\sigma}}\left(\sigma_{i}\right), & \text { for } \sigma_{i} \in \Sigma_{R}(t) \\ \bar{\sigma}, & \text { for } \sigma_{i} \in \Sigma_{N}(t)\end{cases}
$$

and

$$
\frac{\partial W}{\partial t}\left(t, \sigma_{i}(t)\right)=0
$$

The condition in (59) can be rewritten using

$$
\frac{d}{d t}\left(W\left(t, \sigma_{i}(t)\right)\right)=\frac{\partial W}{\partial \sigma} \dot{\sigma}_{i}+\frac{\partial W}{\partial t}=0
$$

Consequently,

$$
\frac{\partial W}{\partial \sigma}\left(t, \sigma_{i}\right)= \begin{cases}\tau_{\bar{\sigma}}^{\prime}\left(\sigma_{i}\right), & \text { for } \sigma_{i} \in \Sigma_{R}(t), \\ 0, & \text { for } \sigma_{i} \in \Sigma_{N}(t) .\end{cases}
$$

This can also be regarded as an interpolation problem, here with multiplicity in $\sigma_{i}$, as is illustrated in Figure 3.

The template function, $\tau_{\bar{\sigma}}$, is here assumed to be time-invariant. In order to improve the bound, time-varying template functions could also be considered, for instance assuming that $\frac{\partial \tau_{\bar{\sigma}}}{\partial t}=0$.

Now, let us assume that we want to reduce a second-order system to first order. The system has two, time-varying, singular values, $\sigma_{1}>\sigma_{2} \geq 0$. Thus, $\Sigma_{R}=\left\{\sigma_{1}\right\}$ and $\Sigma_{N}=\left\{\sigma_{2}\right\}$. This gives the following error bound

$$
\left\|G_{2}-\hat{G}_{1}\right\|=\max _{t} f\left(\frac{\sigma_{2}}{\sigma_{1}}\right) \sigma_{2}
$$

where the amplification factor, $f$, is shown in Figure 4.

If we revisit the diesel exhaust catalyst model from [14], we can compute an upper bound for reducing the 24 th-order model to first order to $3.53 \times 6 \times 10^{-3} \approx 21 \times 10^{-3}$. Note that this bound does not depend on the number of minima in $\sigma_{2}$, but it is instead based on the assumption that $\sigma_{2} / \sigma_{1} \leq 0.1$ holds.

In the general case, assuming the fixed template function (56), we can express an upper bound on the model error as $\left\|G-\hat{G}_{r}\right\| \leq \gamma=\max _{t} F\left(\Sigma_{N}(t), \Sigma_{R}(t)\right)$, where $F$ is defined as the minimum value, $\bar{\sigma}$ such that there exists a PROI function satisfying (58) and (60). 


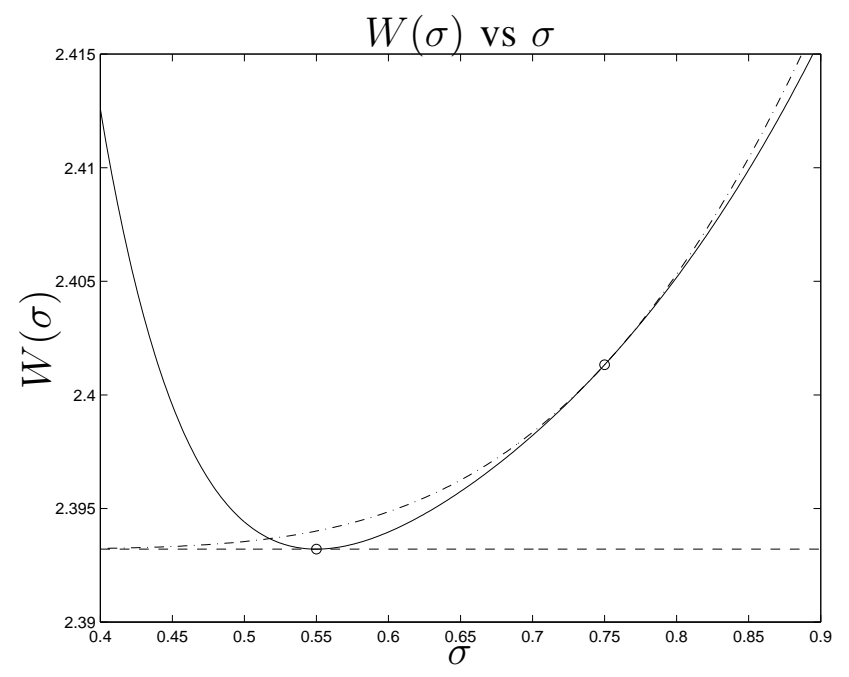

Fig. 3. Interpolation problem for a continuous-time, second-order systems to be reduced to first order. Here $\sigma_{2}(t)=0.75$, and $\sigma_{1}(t)=0.55$, which yields $\bar{\sigma}=2.3932$ as minimum. The function (solid line) tangents the template function (dash-dotted) line at $\sigma=0.75$ and tangents the line $W=\bar{\sigma}$ (dashed) at $\sigma=0.55$.

\section{Discussion}

When trying to find a constant $\bar{\sigma}$ for a reduced order model using the template function in (56), we need to assume that the sets of reduced and remaining singular values are separated. If they go together at some time instant, $t$, such that $\sigma_{r}(t)=\sigma_{r+1}(t)$, the amplification factor will increase infinitely, see Figure 4. If they meet, we need to determine a constant $\bar{\sigma}$ bound as if $\sigma_{r}$ belong to the set of removed singular values. If that $\sigma_{r}(t)$ in turn joins $\sigma_{r-1}$, we have to include that as well, and so on, until the reduced and remaining singular values become separated.

Another related problem is that the upper bound obtained from the template method depends on the number, $r$, of remaining Hankel singular values, even if they are far away from the removed ones. Whether these phenomena are artifacts of the method and its template function is not yet fully understood. Using time-varying template functions would probably produce a less conservative bound, but at an increased computational complexity. Other time-invariant template functions could also be considered but this has yet not been studied.

\section{CONCLUSIONS}

In this paper we present a powerful tool for deriving error bounds for reduced order models of LTV systems. The analysis is based on the Hankel singular values without any further explicit 


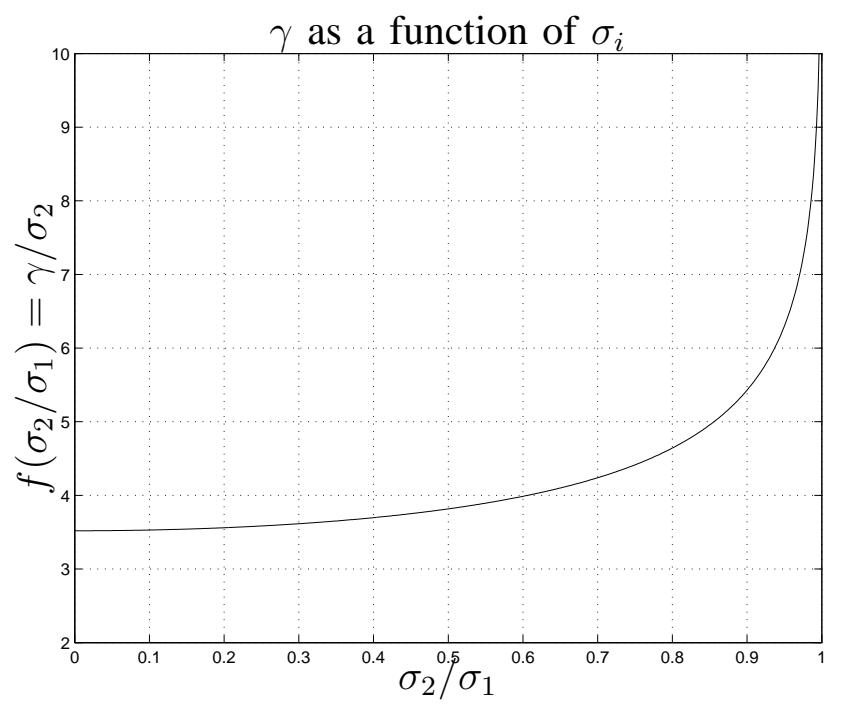

Fig. 4. Amplification factor, $f\left(\sigma_{2} / \sigma_{1}\right)=\gamma / \sigma_{2}$, with $n=2$ and $r=1$ for some different values of $\sigma_{2} / \sigma_{1}$. The template function given in (56) has been used to compute tha amplification factor, which can be approximated as $f(x)=3.5191+$ $3.0165\left(\frac{\operatorname{atanh} x}{x}-1\right)$.

knowledge of the system. By applying a certain class of transformation functions on the Hankel singular values, we provide upper error bounds of the model error.

Two kind of methods are presented: bounds for finite-horizon model errors and bounds for infinite-horizon systems including periodic systems. The choice of method depends mainly on the time-horizon of the problem. In the finite-horizon case, the bound depends on the time-horizon. We can express an upper bound that is a slowly increasing function of the number of minima in the Hankel singular values to be removed. In the infinite-horizon case the model error bound depends on both the removed and remaining Hankel singular values.

We have assumed that all Hankel singular value are strictly greater than zero. However, the theory can be extended to also include the case when they become zero. In addition, it is also straightforward to include the case when the system order is time-varying.

\section{REFERENCES}

[1] B. C. Moore, "Principal component analysis in linear systems: Controllability, observability, and model reduction," IEEE Transactions on Automatic Control, vol. 26, pp. 17-32, 1981.

[2] L. Pernebo and L. Silverman, "Model reduction via balanced state space representation," IEEE Transactions on Automatic Control, vol. 27, no. 2, pp. 382-387, April 1982. 
[3] D. Enns, "Model reduction with balanced realizations: An error bound and a frequency weighted generalization," in IEEE Proceedings of the 23rd Conference on Decision and Control, Las Vegas, Nevada, 1984.

[4] K. Glover, "All optimal Hankel-norm approximations of linear multivariable systems and their $L^{\infty}$-error bounds," International Journal of Control, vol. 39, no. 6, pp. 1115-1193, 1984.

[5] U. Al-Saggaf and G. Franklin, "An error-bound for a discrete reduced-order model of a linear multivariable system," IEEE Transactions on Automatic Control, vol. 32, no. 9, pp. 815-819, September 1987.

[6] D. Hinrichsen and A. J. Pritchard, "An improved error estimate for reduced-order models of discrete-time systems," IEEE Transactions on Automatic Control, vol. 35, pp. 317-320, March 1990.

[7] C. Beck, J. Doyle, and K. Glover, "Model reduction of multi-dimensional and uncertain system," IEEE Transactions on Automatic Control, vol. 41, no. 10, pp. 1466-1477, October 1996.

[8] D. Kavranoğlu and M. Bettayeb, "Characterization of the solution to the optimal $H_{\infty}$ model reduction problem," Systems \& Control Letters, vol. 20, no. 2, pp. 99-107, February 1993.

[9] D. Kavranoğlu, "A computational scheme for $H_{\infty}$ model reduction," IEEE Transactions on Automatic Control, vol. 39, no. 7, pp. 1447-1451, July 1994.

[10] S. Lall and C. Beck, "Error bounds for balanced model-reduction of linear time-vaying systems," IEEE Transactions on Automatic Control, vol. 48, no. 6, pp. 946-956, June 2003.

[11] N. Cohen and I. Lewkowicz, "Convex invertible cones and the Lyapunov equation," Linear Algebra and its Applications, vol. 250, pp. 105-131, 1997.

[12] S. Shokoohi, L. Silverman, and P. V. Dooren, "Linear time-variable systems: Balancing and model reduction," IEEE Transactions on Automatic Control, vol. 28, no. 8, pp. 714-724, August 1983.

[13] S. Shokoohi and L. Silverman, "Identification and model reduction of time-varying discrete-time systems," Automatica, vol. 23, no. 4, pp. 509-521, 1987.

[14] H. Sandberg and A. Rantzer, "Balanced truncation of linear time-varying systems," IEEE Transactions on Automatic Control, vol. 49, no. 2, pp. 217-229, February 2004.

[15] F. R. Gantmacher, The Theory of Matrices. New York: Chelsea Publishing Company, 1959, 1971, vol. II.

[16] N. Cohen and I. Lewkowicz, "Convex invertible cones, nevanlinna-pick interpolation and the set of lyapunov solutions," in Proceedings 15th International Symposium on Mathematical Theory of Networks and Systems, 2002, pp. 1-6.

[17] S. Lall, C. Beck, and G. Dullerud, "Guaranteed error bounds for model reduction of linear time-varying systems," in Proceedings of the American Control Conference, Philadelphia, Pennsylvania, 1998, pp. 634-638.

[18] S. Longhi and G. Orlando, "Balanced reduction of linear periodic systems," Kyberniteka, vol. 35, no. 6, pp. 737-751, 1999.

[19] A. Varga, "Balanced truncation model reduction of periodic systems," in Proceedings of the 39th IEEE Conference on Decision and Control, Sydney, Australia, 2000, pp. 2379-2384.

[20] A. Blomqvist and R. Nagamune, "An extension of a Nevanlinna-Pick interpolation solver to cases including derivative constraints," in IEEE Proceedings of the 41st Conference on Decision and Control, Las Vegas, Nevada, December 2002, pp. 2552-2557.

[21] T. Georgiou, "Analytic interpolation and the degree constraint," Int. J. Applied Mathematics and Computer Science, vol. 11, no. 3, pp. 101-109, March 2001.

[22] A. Helmersson, "Model reduction using LMIs," in Proceedings of the 33rd Conference on Decision and Control, vol. 4, Lake Buena Vista, Florida, December 1994, pp. 3217-3222. 